\title{
Psoriasis severity and hypothalamic- pituitary-adrenal axis function: results from the CALIPSO study
}

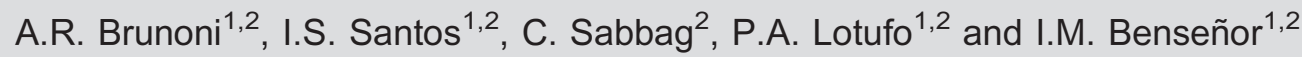 \\ ${ }^{1}$ Centro de Pesquisa Clínica e Epidemiológica, Hospital Universitário, Universidade de São Paulo, São Paulo, SP, Brasil \\ ${ }^{2}$ Faculdade de Medicina, Universidade de São Paulo, São Paulo, SP, Brasil
}

\begin{abstract}
Psoriasis is a chronic inflammatory disease that significantly impacts life quality, being associated with stress and mental disorders. We investigated whether the activity of the hypothalamic-pituitary-adrenal (HPA) axis was associated with psoriasis severity, daily life stress and anxiety, and depressive symptoms. In this ancillary study, which was part of the CALIPSO (coronary artery calcium in psoriasis) study, saliva was collected from 102 patients with psoriasis immediately upon awakening, 30 , and $60 \mathrm{~min}$ after awakening, at 2:00 pm and at bedtime (five time points) to determine salivary cortisol levels. We used Pearson's correlation coefficient to evaluate the association of clinical and psychopathological variables with HPA activity. We found a direct correlation between bedtime cortisol and psoriasis severity evaluated by the psoriasis area severity index (PASI; $r=0.39, P<0.001$ ). No correlations between other clinical and psychopathological variables or with other cortisol assessments were observed. The findings indicated that HPA dysfunction may be present in psoriasis, as bedtime cortisol was correlated with psoriasis severity. Our study is limited by the lack of a control group; therefore, we were not able to explore whether these cortisol values were different compared with a concurrent, healthy sample.
\end{abstract}

Key words: Psoriasis; Cortisol; HPA function; Clinical study

\section{Introduction}

Psoriasis is a chronic inflammatory disease with a prevalence of up to $2-4 \%$ in the adult population $(1,2)$. Although most presentations are mild and limited to localized skin lesions, severe cases present extensive skin and joint involvement and have an important impact on life quality. In fact, patients with psoriasis present an increased prevalence of mental disorders, such as major depression (3). Moreover, exacerbations of psoriasis episodes are often preceded by stressful life episodes (4). The physiological responses to stress include increased sympathetic activity, over-activation of the hypothalamic-pituitary-adrenal (HPA) axis, and release of pro-inflammatory cytokines, which can, in turn, perpetuate and aggravate psoriasis, as the disease is associated with increased activity of several pro-inflammatory cytokines (5).

Psychological stress thus seems to be an important, bidirectional link between psoriasis, decreased life quality and mental disorders, and within that context, over-activity of the HPA axis is one of the physiological factors associated with stress and stress response. HPA activity can be indexed by measuring cortisol levels, but only a few studies have evaluated the role of cortisol in psoriasis, and they offer mixed results. For instance, one early study (6) compared the serum cortisol levels at rest and after a stress test in 10 patients with psoriasis with the levels in 10 healthy subjects. The authors found no difference with cortisol levels at rest, but the decrease in cortisol levels after a stress test was greater in patients with psoriasis, suggesting an involvement of the HPA axis in these patients. The inconsistency of published results could be associated with the use of cortisol levels in the blood, which vary markedly during the day (7). With the advancement of methods evaluating cortisol levels in the saliva (8), new variables to measure the activity of the HPA axis become possible, such as the evaluation of the daily salivary cortisol curve or the levels of cortisol immediately after awakening or at bedtime (7). Nevertheless, the role of salivary cortisol in psoriasis has not been adequately investigated.

Therefore, the aim of this study was to investigate the association of stress with psoriasis through the assessment

Correspondence: A.R. Brunoni, Centro de Pesquisa Clínica e Epidemiológica, Hospital Universitário, USP, Av. Lineu Prestes 2565, 3으 andar, 05508-000 São Paulo, SP, Brasil. Fax: +55-11-3091-9341. E-mail: brunoni@usp.br 
of several psychopathological and clinical variables and the daily salivary cortisol curve.

\section{Material and Methods}

\section{Subjects}

This ancillary study was part of the CALIPSO (coronary artery calcium in psoriasis) study, which investigated subclinical atherosclerosis, clinical coronary heart disease, and cardiovascular risk factors in adult (older than 35 and 40 years for men and women, respectively) patients who presented psoriasis of more than 3 years duration. As this ancillary study started while the CALIPSO study was ongoing, only the last 119 participants were invited to participate. The Internal Review Board approved the study, and all participants signed an informed consent.

\section{Assessments}

An expert dermatologist in a referral outpatient clinic recruited all cases of psoriasis. For each participant, the standardized psoriasis area severity index (PASI) (9) was calculated to estimate disease involvement, with higher scores indicating greater severity. The Dermatological Life Quality Index (DLQI) (10) was used to assess the impact of the condition on quality of life. Scores were classified as none (0 to 1 , no effect at all on patient's life); mild (scores from 2 to 5 ); moderate (6 to 10 ); severe (11 to 20 ) and very severe (21 to 30 , indicating an extreme effect on patient's life). The State-Trait Anxiety Inventory (STAI) questionnaire was used to evaluate anxiety traits in the patients (11), with higher scores indicating subjects with more anxiety traits. We also used the Patient Health Questionnaire (PHQ-9) (11), a nine-item summed scale with scores ranging from 0 (no depressive symptoms) to 27 (all depressive symptoms occurring daily) to evaluate the severity of depressive symptoms in our sample. Finally, the Holmes and Rahe scale (12) was used to assess the total "load" of stressful life events 2 years prior to the interview. Higher scores in this scale mean a greater quantity and/or quality of stressful life events.

All participants answered an extensive questionnaire asking about previous and family history of hypertension, diabetes, dyslipidemia, coronary heart disease, angina, myocardial infarction, revascularization (percutaneous or surgical), occupational history, socioeconomic status, education, smoking, physical activity, and psychiatric disorders. Anthropometric parameters (weight, height, sitting height, and waist and hip circumferences) were measured using standard equipment and techniques. Blood samples were collected after a 12-h overnight fast. A standard $75 \mathrm{~g}$ oral glucose tolerance test was administered to all participants without a previous diagnosis of diabetes. Hypertension was defined as the presence of one of the following: a) medical history of hypertension, b) use of antihypertensive treatment, c) systolic blood pressure $\geq 140 \mathrm{mmHg}$, or d) diastolic blood pressure $\geq 90 \mathrm{mmHg}$. Diabetes was defined as the presence of one of the following: a) medical history of diabetes, b) use of oral hypoglycemic treatment or insulin, c) fasting plasma glucose $\geq 126 \mathrm{mg} / \mathrm{dL}$, or d) 2-h oral glucose tolerance test $\geq 200 \mathrm{mg} / \mathrm{dL}$.

The laboratory tests included fasting plasma glucose and plasma glucose $120 \mathrm{~min}$ after an overload of $75 \mathrm{~g}$ of glucose (oral glucose tolerance test, OGTT); total cholesterol and HDL-cholesterol by an enzymatic colorimetric assay; triglycerides by an enzymatic colorimetric assay (glycerol phosphate peroxidase); LDL-cholesterol calculated by the Friedewald equation except in participants with triglycerides $>400 \mathrm{mg} / \mathrm{dL}$, in whom LDL-cholesterol was determined by an enzymatic colorimetric assay; creatinine by an enzymatic colorimetric assay (Jaffé; all from ADVIA Chemistry, Siemens, Germany); glycated hemoglobin $\left(\mathrm{HbA}_{1}\right)$ by high pressure liquid chromatography, Bio-Rad, USA; and microalbuminuria using an immunochemical assay (nefelometry, Dade Bhering, Siemens, Germany).

Saliva samples were collected using Salivettes ${ }^{\circledR}$ (Sarstedt, Germany) with an insert containing a sterile polyester swab, which yields a clear, particle-free sample. Trained staff instructed patients to collect five salivary cortisol samples (within 5 min of awakening; 30 and 60 min after awakening; at 2:00 pm or after lunch, whichever was earlier; and 5 min before going to bed). To avoid meal-related surges in cortisol, which can falsely increase cortisol values, subjects were told to avoid brushing their teeth or eating 30 min prior to each sample collection. To increase compliance to the instructions, participants were told to write down the time when the samples were collected. Samples were excluded from analysis if the time of collection deviated by more than 5 min for the first 3 samples (i.e., salivary samples with the first hour after awakening) or by $15 \mathrm{~min}$ for the remaining samples. Less than $5 \%$ of the samples were excluded for this reason. The time of each collection was self-reported by the patients.

The salivary samples were delivered to our research center by expedited postal mail (maximum of 5 days after collection); participants were instructed to refrigerate the Salivettes after each collection. When they arrived at the research center, Salivettes were centrifuged at $250 \mathrm{~g}$ for $15 \mathrm{~min}$, and the filtrates were stored frozen at $-20^{\circ} \mathrm{C}$ until analysis by ELISA. Before analysis, the samples were thawed and individually recentrifuged ( $250 \mathrm{~g}$ for $15 \mathrm{~min}$ ). Cortisol levels were determined using a commercially available ELISA (Diametra, Italy). Intra- and interassay coefficients of variation were lower than $8 \%$, which was within the value indicated for this ELISA kit. The lowest concentration detectable by the kit is $0.05 \mathrm{ng} / \mathrm{mL}$ and the highest is $100 \mathrm{ng} / \mathrm{mL}$. Also, although there are no normal values for salivary cortisol levels, the suggested normal range, according to the kit manufacturer, is 3-10 for morning and $0.6-2.5 \mathrm{ng} / \mathrm{mL}$ for afternoon measurements (a suggested range was not provided for values measured during the evening). 


\section{Statistical analysis}

Categorical variables are reported as number and proportion; continuous variables are reported as means and standard deviations. Pearson's correlation coefficients, $t$-tests, or one-way analysis of variance (ANOVA) were performed to explore the association of salivary cortisol with psychopathological variables.

We assessed the correlations of the following cortisol measurements with cardiovascular and psychopathological characteristics: 1) the first daily sample, corresponding to the "wake-up value"; 2) the cortisol awakening response (CAR), corresponding to the difference between the second and the first sample; 3 ) the area under the curve (AUC) corresponding to the first hour (i.e., first 3 salivary assessments); 4) the AUC of the complete day (all samples); and 5) the "bedtime value", corresponding the last salivary cortisol collection. The time of each collection was used to estimate the AUCs using the trapezoidal rule as described elsewhere (13). Briefly, this formula estimates the AUC by calculating the mean of 2 consecutive cortisol measurements, which is then multiplied by the time interval between them. The scores from each time interval are then summed in order to obtain the AUC.

\section{Results}

We recruited 119 participants for this ancillary study, and $17(14.3 \%)$ who agreed to participate did not send salivary samples to the central laboratory. Data from the remaining 102 patients ( $51 \%$ females) who returned the 5 salivary samples collected during the day to the laboratory were included in the analysis. None of the included patients were using corticosteroid drugs. The baseline characteristics of the sample are reported in Table 1. The raw and derived cortisol measurements of the sample are reported in Table 2.

\section{Salivary cortisol and clinical and demographic variables}

We found correlations between 1) awakening cortisol and bedtime cortisol, CAR and the AUC of the first hour, 2) the AUC of the first hour and AUC of the day, and 3) STAI and depressive symptoms. These associations were as expected and confirmed the proper collection of the cortisol samples evaluated in our study.

No correlations between salivary cortisol values and continuous psychopathological variables were found (Table 3) except for a positive correlation $(r=0.39$, $\mathrm{P}<0.001$ ) between salivary cortisol values at bedtime and the PASI score of the extent and severity of psoriasis, i.e., higher cortisol values were associated with a greater severity of psoriasis.

No correlations were found between salivary cortisol values and the DLQI when analyzed as a categorical variable $(\mathrm{Fs}<1.6, \mathrm{Ps}>0.14)$.
Table 1. Clinical, psychopathological and demographic variables of 102 patients with psoriasis.

\begin{tabular}{lc}
\hline Variable & \\
\hline Male/female (\%) & $50 / 52(49 / 51 \%)$ \\
Age $($ mean \pm SD) & $58 \pm 8.2$ \\
BMI, $\mathrm{kg} / \mathrm{m}^{2}($ mean $\pm \mathrm{SD})$ & $30.4 \pm 6.65$ \\
Diabetes mellitus, $\mathrm{n}(\%)$ & $23(22.5 \%)$ \\
Hypertension, $\mathrm{n}(\%)$ & $45(44 \%)$ \\
Psoriasis Area Severity Index (mean $\pm \mathrm{SD})$ & $9.1 \pm 8.5$ \\
STAl (mean $\pm \mathrm{SD})$ & $39.6 \pm 11.1$ \\
PHQ-9 (mean $\pm \mathrm{SD})$ & $6.4 \pm 5.9$ \\
Depression, $\mathrm{n}(\%)$ & $21(20.6 \%)$ \\
Effect of psoriasis on life quality, $\mathrm{n}(\%)$ & \\
$\quad$ No effect $(0-1)$ & $22(21.6 \%)$ \\
$\quad$ Mild effect (2-5) & $40(39.2 \%)$ \\
$\quad$ Moderate effect (6-10) & $20(19.6 \%)$ \\
Severe effect (11-20) & $13(12.7 \%)$ \\
Very severe effect $(21-30)$ & $7(6.9 \%)$ \\
\hline
\end{tabular}

Effect of psoriasis on life quality was determined according to the Dermatological Life Quality Index (DLQI) score. BMI: body mass index; SD: standard deviation; STAI: State-Trait Anxiety Inventory; PHQ-9: Patient Health Questionnaire.

\section{Discussion}

In this sample of 102 patients with psoriasis, we evaluated the association of several clinical and psychopathological variables with salivary cortisol values measured throughout the day. Our main finding was that the salivary cortisol level at bedtime was directly correlated with psoriasis severity as measured by the Psoriasis Area Severity Index. We did not find any significant correlations between other variables, which included those measuring depressive and anxiety symptoms, stressful life events, life quality, and salivary cortisol.

The significant correlation between bedtime cortisol and psoriasis severity is in agreement with literature showing

Table 2. Cortisol values observed for 102 patients with psoriasis.

\begin{tabular}{lc}
\hline Assessment & Cortisol $(\mathrm{ng} / \mathrm{mL})$ \\
\hline 1st (wake-up) & $4.97 \pm 3$ \\
2nd (+30 min) & $6.77 \pm 3.66$ \\
3rd (+60 min) & $5.5 \pm 4.28$ \\
4th (2:00 pm) & $3.13 \pm 4.19$ \\
5th (bedtime) & $1.33 \pm 1.67$ \\
Cortisol awakening response & $1.82 \pm 3.36$ \\
AUC-1 h & $6.01 \pm 2.76$ \\
AUC-day & $50.85 \pm 45.24$ \\
\hline
\end{tabular}

Data are reported as means \pm SD. AUC, area under the curve. AUC-1 $\mathrm{h}$ and AUC-day represent the AUC during the first hour and the entire day, respectively. 
Table 3. Pearson's correlations between psychopathological and salivary cortisol values.

\begin{tabular}{|c|c|c|c|c|c|c|c|c|c|c|}
\hline & \multicolumn{10}{|c|}{ Pearson's correlations } \\
\hline & $\mathrm{A} 1$ & A5 & CAR & AUC-1 & AUC-day & DLQI & PHQ-9 & STAI & HOLMES & PASI \\
\hline $\mathrm{A} 1$ & 1 & & & & & & & & & \\
\hline A5 & $0.291^{* *}$ & 1 & & & & & & & & \\
\hline CAR & $0.331^{* *}$ & 0.073 & 1 & & & & & & & \\
\hline$A \cup C-1$ & $0.633^{* *}$ & $0.235^{*}$ & $-0.431^{\star *}$ & 1 & & & & & & \\
\hline AUC-day & 0.053 & 0.151 & -0.059 & $0.432^{\star *}$ & 1 & & & & & \\
\hline DLQI & -0.096 & 0.136 & -0.160 & 0.020 & -0.022 & 1 & & & & \\
\hline PHQ-9 & -0.035 & 0.057 & -0.034 & 0.004 & -0.132 & $0.336^{\star *}$ & 1 & & & \\
\hline STAI & -0.023 & 0.086 & -0.053 & 0.025 & 0.011 & $0.268^{\star}$ & $0.630^{\star *}$ & 1 & & \\
\hline HOLMES & -0.086 & -0.054 & -0.143 & 0.043 & -0.042 & 0.037 & -0.001 & 0.087 & 1 & \\
\hline PASI & 0.12 & $0.39^{* *}$ & 0.14 & 0.03 & 0.09 & 0.1 & -0.06 & 0.03 & -0.02 & 1 \\
\hline
\end{tabular}

Bold values indicate significant associations at ${ }^{*} \mathrm{P}<0.01$ or ${ }^{*} \mathrm{P}<0.05$. CAR: cortisol awakening response; AUC: area under the curve; DLQI: Dermatological Life Quality Index; PHQ-9: Patient Health Questionnaire; STAI: State-Trait Anxiety Inventory; HOLMES: Holmes and Rahe Stressful Life Events Scale; PASI: Psoriasis Area Severity Index. Values from A1 and A5 represent the 1st cortisol assessment (wake-up), and the 5th cortisol assessment (at bedtime), respectively. AUC-1 and AUC-day represent the AUC during the first hour and the entire day, respectively.

that bedtime cortisol has the highest between-visit reliability of all the measurements of daily cortisol (14), and has a good correlation with adrenal gland volume. Interestingly, one study (15) identified an association between bedtime cortisol and decreased prefrontal cortical volumes in another model of chronic stress (posttraumatic stress symptoms) in children. As the prefrontal cortex down-regulates the HPA axis, such a finding could also explain the association between psoriasis severity and bedtime cortisol. One important point is that the PASI was assessed within the same week as cortisol levels, thus reflecting the disease activity that was associated with salivary cortisol levels.

Previous findings also corroborate the association between psoriasis severity and cortisol expression. In one study, Amatya et al. (16) reported a correlation between activity in biopsies of lesion sites in patients with psoriasis (measured by the presence of neuroinflammatory cells) and salivary cortisol. Other studies $(17,18)$ reported a correlation between psoriasis and cortisol levels; however, they found hypocortisolism in patients with psoriasis and high stress. Although they appear to be contradictory, those studies and ours report HPA dysfunction as a physiological finding in psoriasis. Moreover, those studies found that hypocortisolism was associated with perceived stress and not, as observed in our study, directly associated with psoriasis severity. Although we did not find an association between stress and cortisol levels in our study sample, that could be related in part to the instrument used to assess stressful life events, as the Holmes and Rahe scale attributes the same stress score to several life events that might be experienced differently by each person. However, the studies that observed hypocortisolism either addressed stressful life events prospectively (18) or measured cortisol levels after a stress-induced task (17).
Our study has several limitations. First, we did not recruit a control group because this was an ancillary study with participants recruited from within a larger study evaluating cardiovascular risk factors and subclinical atherosclerosis in patients with psoriasis. The normal ranges of morning and afternoon cortisol values suggested by the assay kit manufacturer (3-10 and 0.6-2.5 ng/mL, respectively) were used, although they were described as a "preliminary guideline until each laboratory establishes its own normal range". Therefore, it would be necessary to compare salivary cortisol values of psoriasis patients with healthy volunteers, to identify any differences. Second, we did not use electronic monitoring devices to verify saliva collection times, which would have increased reliability compared with self-reporting as used in our study (19). Nevertheless, such increased reliability is particularly important for the CAR, but not as significant for other time periods, especially bedtime cortisol, in which we identified a significant association with psoriasis severity. Third, we observed an association only for bedtime cortisol, but not for other cortisol assessments. Bedtime cortisol was collected $5 \mathrm{~min}$ before going to bed, and presented less variation than other assessments, for example measurements of the afternoon cortisol levels, probably because it is easier to incorporate collection of bedtime salivary cortisol in the daily routine (20); it presents important variation considering the bedtime hour itself as it does not use a fixed hour. In addition, we did not find an association between the daily AUC, which takes differences in the time of collection into account, with other psoriasis indexes. Fourth, several analyses were performed. We explored the correlation of five cortisol variables (i.e., collection data) with five clinical-demographic variables. Even taking these 25 tests into account, the correlation would still be 
significant following application of the Bonferroni test, as the resulting $P$ value would be 0.002 ( 0.05 divided by 25); we found a $\mathrm{P}<0.001$.

\section{References}

1. Gelfand JM, Gladman DD, Mease PJ, Smith N, Margolis DJ, Nijsten $\mathrm{T}$, et al. Epidemiology of psoriatic arthritis in the population of the United States. J Am Acad Dermatol 2005; 53: 573, doi: 10.1016/j.jaad.2005.03.046.

2. Kurd SK, Gelfand JM. The prevalence of previously diagnosed and undiagnosed psoriasis in US adults: results from NHANES 2003-2004. J Am Acad Dermatol 2009; 60: 218-224, doi: 10.1016/j.jaad.2008.09.022.

3. Dominguez PL, Han J, Li T, Ascherio A, Qureshi AA. Depression and the risk of psoriasis in US women. J Eur Acad Dermatol Venereol 2013; 27: 1163-1167, doi: 10.1111/j. 1468-3083.2012.04703.x.

4. Rampton DS. The influence of stress on the development and severity of immune-mediated diseases. J Rheumatol Suppl 2011; 88: 43-47, doi: 10.3899/jrheum.110904.

5. Basavaraj $\mathrm{KH}$, Navya MA, Rashmi R. Stress and quality of life in psoriasis: an update. Int J Dermatol 2011; 50: 783792, doi: 10.1111/j.1365-4632.2010.04844.x.

6. Arnetz BB, Fjellner B, Eneroth P, Kallner A. Stress and psoriasis: psychoendocrine and metabolic reactions in psoriatic patients during standardized stressor exposure. Psychosom Med 1985; 47: 528-541, doi: 10.1097/00006842198511000-00003.

7. Hellhammer DH, Wust S, Kudielka BM. Salivary cortisol as a biomarker in stress research. Psychoneuroendocrinology 2009; 34: 163-171, doi: 10.1016/j.psyneuen.2008.10.026.

8. Kirschbaum C, Hellhammer DH. Salivary cortisol in psychoneuroendocrine research: recent developments and applications. Psychoneuroendocrinology 1994; 19: 313-333, doi: 10.1016/0306-4530(94)90013-2.

9. Finlay AY. Current severe psoriasis and the rule of tens. $\mathrm{Br} \mathrm{J}$ Dermatol 2005; 152: 861-867, doi: 10.1111/j.1365-2133.2005. 06502.x.

10. Finlay AY, Khan GK. Dermatology Life Quality Index (DLQI) a simple practical measure for routine clinical use. Clin Exp Dermatol 1994; 19: 210-216, doi: 10.1111/j.1365-2230.1994. tb01167.x

11. Gorenstein C, Andrade LHSG, Zuardi AW. Escalas de avaliação clínica em psiquiatria e psicofarmacologia. São Paulo: Lemos Editorial; 2000.
We conclude that HPA dysfunction may be associated with psoriasis, as bedtime cortisol values were correlated with psoriasis severity.

12. Holmes $\mathrm{TH}$, Rahe RH. The Social Readjustment Rating Scale. J Psychosom Res 1967; 11: 213-218, doi: 10.1016/ 0022-3999(67)90010-4.

13. Pruessner JC, Kirschbaum C, Meinlschmid G, Hellhammer $\mathrm{DH}$. Two formulas for computation of the area under the curve represent measures of total hormone concentration versus time-dependent change. Psychoneuroendocrinology 2003; 28: 916-931, doi: 10.1016/S0306-4530(02)00108-7.

14. Golden SH, Wand GS, Malhotra S, Kamel I, Horton K. Reliability of hypothalamic-pituitary-adrenal axis assessment methods for use in population-based studies. Eur J Epidemiol 2011; 26: 511-525, doi: 10.1007/s10654-011-9585-2.

15. Carrion VG, Weems CF, Richert K, Hoffman BC, Reiss AL. Decreased prefrontal cortical volume associated with increased bedtime cortisol in traumatized youth. Biol Psychiatry 2010; 68: 491-493, doi: 10.1016/j.biopsych.2010. 05.010 .

16. Amatya B, El-Nour H, Holst M, Theodorsson E, Nordlind K. Expression of tachykinins and their receptors in plaque psoriasis with pruritus. Br J Dermatol 2011; 164: 1023-1029, doi: 10.1111/j.1365-2133.2011.10241.x.

17. Richards HL, Ray DW, Kirby B, Mason D, Plant D, Main CJ, et al. Response of the hypothalamic-pituitary-adrenal axis to psychological stress in patients with psoriasis. Br J Dermatol 2005; 153: 1114-1120, doi: 10.1111/j.1365-2133.2005.06817.x.

18. Evers AW, Verhoeven EW, Kraaimaat FW, de Jong EM, de Brouwer SJ, Schalkwijk J, et al. How stress gets under the skin: cortisol and stress reactivity in psoriasis. $\mathrm{Br} J$ Dermatol 2010; 163: 986-991, doi: 10.1111/j.1365-2133.2010.09984.x.

19. Kudielka BM, Broderick JE, Kirschbaum C. Compliance with saliva sampling protocols: electronic monitoring reveals invalid cortisol daytime profiles in noncompliant subjects. Psychosom Med 2003; 65: 313-319, doi: 10.1097/01.PSY.0000058374. 50240.BF.

20. Golden SH, Sanchez BN, Wu M, Champaneri S, Diez Roux $A V$, Seeman $T$, et al. Relationship between the cortisol awakening response and other features of the diurnal cortisol rhythm: the Multi-Ethnic Study of Atherosclerosis. Psychoneuroendocrinology 2013; 38: 2720-2728, doi: 10.1016/j.psyneuen.2013.06.032. 